\title{
On zeros of irreducible characters lying in a normal subgroup
}

\section{J. Felipe, N. Grittini \& V. Sotomayor}

Annali di Matematica Pura ed

Applicata (1923 -)

ISSN 0373-3114

Annali di Matematica

DOI 10.1007/s10231-020-00942-1 
Your article is protected by copyright and all rights are held exclusively by Fondazione Annali di Matematica Pura ed Applicata and Springer-Verlag GmbH Germany, part of Springer Nature. This e-offprint is for personal use only and shall not be selfarchived in electronic repositories. If you wish to self-archive your article, please use the accepted manuscript version for posting on your own website. You may further deposit the accepted manuscript version in any repository, provided it is only made publicly available 12 months after official publication or later and provided acknowledgement is given to the original source of publication and a link is inserted to the published article on Springer's website. The link must be accompanied by the following text: "The final publication is available at link.springer.com". 


\title{
On zeros of irreducible characters lying in a normal subgroup
}

\author{
M. J. Felipe ${ }^{1} \mathbb{D} \cdot$ N. Grittini ${ }^{2} \cdot$ V. Sotomayor ${ }^{1}$ (D)
}

Received: 28 July 2019 / Accepted: 11 January 2020

(c) Fondazione Annali di Matematica Pura ed Applicata and Springer-Verlag GmbH Germany, part of Springer Nature 2020

\begin{abstract}
Let $N$ be a normal subgroup of a finite group $G$. In this paper, we consider the elements $g$ of $N$ such that $\chi(g) \neq 0$ for all irreducible characters $\chi$ of $G$. Such an element is said to be non-vanishing in $G$. Let $p$ be a prime. If all $p$-elements of $N$ satisfy the previous property, then we prove that $N$ has a normal Sylow $p$-subgroup. As a consequence, we also study certain arithmetical properties of the $G$-conjugacy class sizes of the elements of $N$ which are zeros of some irreducible character of $G$. In particular, if $N=G$, then new contributions are obtained.
\end{abstract}

Keywords Finite groups $\cdot$ Normal subgroups $\cdot$ Irreducible characters $\cdot$ Conjugacy classes

Mathematics Subject Classification $20 \mathrm{C} 15 \cdot 20 \mathrm{E} 45$

\section{Introduction}

In the sequel, all groups considered are finite. Within character theory, a classical theorem of Burnside asserts that a nonlinear irreducible character of a finite group always vanishes on some element. It is not difficult to see that the converse is also true, so the rows of the character table of a group that contain a zero entry are completely characterised. However, the "dual" situation for conjugacy classes fails in general: a column that corresponds to a non-central conjugacy class may not contain a zero. This fact somehow violates the standard duality that in many cases arises between irreducible characters and conjugacy classes

V. Sotomayor

vicorso@doctor.upv.es

M. J. Felipe

mfelipe@mat.upv.es

N. Grittini

nicola.grittini@unifi.it

1 Instituto Universitario de Matemática Pura y Aplicada (IUMPA), Universitat Politècnica de València, Camino de Vera, s/n, 46022 Valencia, Spain

2 Dipartimento di Matematica U. Dini, Università degli Studi di Firenze, Viale Morgagni 67/a, 50134 Florence, Italy 
of a group. Therefore, for a group $G$, an element $g$ is said to be non-vanishing in $G$ if $\chi(g) \neq 0$ for every irreducible character $\chi$ of $G$.

An immediate corollary to the aforementioned Burnside's result is that a group is abelian if and only if every element is non-vanishing. Isaacs et al. [10] obtained elegant results about the location of non-vanishing elements in certain groups. For example, for a nilpotent group $G$, an element is non-vanishing if and only if it lies in the centre of $G$. They also proved that if $G$ is soluble, then $g \mathbf{F}(G)$ is a 2-element for a non-vanishing element $g$ of $G$. Consequently, if $g$ is of odd order, then $x$ lies in $\mathrm{F}(G)$. These authors conjectured that every non-vanishing element of a soluble group $G$ lies in $\mathbf{F}(G)$, and it is still an open problem. In this paper, we prove the following result which provides further evidence for this conjecture.

Theorem A Let $N$ be a normal subgroup of a group $G$, and let $p$ be a prime. If $\chi(x) \neq 0$ for every p-element $x \in N$ and for all $\chi \in \operatorname{Irr}(G)$, then $N$ has a normal Sylow p-subgroup.

In particular, if $\chi(x) \neq 0$ for every prime power order element $x \in N$ and for all $\chi \in \operatorname{Irr}(G)$, then $N$ is nilpotent.

Therefore, the arithmetical properties of the non-vanishing elements of $G$ that lie in a normal subgroup $N$ control the structure of $N$. This is interesting since, although we cannot construct the character table of $N$ from the one of $G$, normal subgroups and vanishing elements of $G$ can be easily read from its character table.

Regarding the first claim of Theorem A when $N=G$, we provide extra information on the structure of a $p$-complement of $G$ in Corollary 1, which extends [7, Theorem A]. Concerning the second assertion in Theorem A, when $N=G$, it holds that the group is abelian (see Theorem 1). However, this fact might not happen for the case of a normal subgroup as Example 1 shows.

Actually, we prove Theorem A as a consequence of the next result. We will denote by $1_{G}$ the trivial character of a group $G$.

Theorem B Let $N$ be a normal subgroup of a group $G$, and let $P$ be a Sylow p-subgroup of $G$ for some prime $p$. Let $P_{0}=P \cap N$ and $\beta \in \operatorname{Irr}\left(P / P_{0}\right)$. Then, the following conditions are pairwise equivalent:

(i) $P_{0}$ is a normal Sylow p-subgroup of $N$.

(ii) $\chi(x) \neq 0$ for all irreducible constituents $\chi$ of $\left(1_{P_{0}}\right)^{G}$ and all $x \in P_{0}$.

(iii) $\quad \chi(x) \neq 0$ for all irreducible constituents $\chi$ of $\beta^{G}$ and all $x \in P_{0}$.

Indeed, Theorem B generalises [11, Theorem B] when $N=G$ (see Theorem 3). Notice that, by Theorem 3(i)-(ii), $P_{0}$ is normal in $N$ if and only if $\eta(x) \neq 0$ for all irreducible constituents $\eta$ of $\left(1_{P_{0}}\right)^{N}$ and all $x \in P_{0}$; however, this fact does not directly imply (ii) of Theorem B, nor vice versa. Further, the following equivalence, which is related to Theorem 3(i)-(iii), is not true: $P_{0}$ is normal in $N$ if and only if $p$ does not divide $\chi(1)$ for all irreducible constituents of $\left(1_{P_{0}}\right)^{G}$; it is enough to observe Example 2(2).

As a consequence of Theorem A, some features of a normal subgroup $N$ of a group $G$ can be obtained through the analysis of its $G$-conjugacy class sizes of elements which are zeros of some irreducible character of $G$; such an element is said to be vanishing in $G$. Let $\pi$ be any set of primes, and let $x^{G}$ be the conjugacy class of $x$ in $G$. If $\left|x^{G}\right|$ is a $\pi^{\prime}$-number for every prime power order $\pi$-element $x$ in $N$, then by the main result of Beltrán et al. (see 
[2, Theorem B]), it is known that $N$ has a nilpotent Hall $\pi$-subgroup. In the next result, we show that we do not need to assume this condition for all prime power order $\pi$-elements in $N$, but for those which are vanishing in $G$. However, we need to assume the $\pi$-separability of $N$ to get that result.

Theorem C Let $N$ be a normal subgroup of a group $G$, and let $\pi$ be any set of prime numbers.

(1) Suppose that $\left|x^{G}\right|$ is a $\pi^{\prime}$-number for every prime power order $\pi$-element $x \in N$ which is vanishing in $G$. If $N$ is $\pi$-separable, then $N / \mathbf{O}_{\pi^{\prime}}(N)$ has a nilpotent normal Hall $\pi$ -subgroup. In particular, the Hall $\pi$-subgroups of $N$ are nilpotent.

(2) Suppose that $\left|x^{G}\right|$ is a $\pi$-number for every prime power order $\pi$-element $x \in N$ which is vanishing in $G$. If $\operatorname{Hall}_{\pi}(N) \neq \emptyset$, then $N$ has a normal Hall $\pi$-subgroup. Additionally, if all $\left|x^{G}\right|$ are also $\pi$-numbers for the prime power order $\pi^{\prime}$-elements $x \in N$ that are vanishing in $G$, then the Hall $\pi^{\prime}$-subgroups of $N$ are nilpotent.

We do not know whether the $\pi$-separability condition on $N$ in (1) can be weakened in order to obtain the nilpotency of its Hall $\pi$-subgroups. What is certainly true is that this condition is necessary for the normality of the Hall $\pi$-subgroup of $N / \mathbf{O}_{\pi^{\prime}}(N)$, as Example 4 shows. Additionally, statement (2) above extends for a set of primes the following result proved in [5]: if a prime $p$ does not divide any conjugacy class size of a vanishing $p^{\prime}$-element $x$ of prime power order of a group $G$, then $G$ has a normal $p$-complement. We do not know whether the assumption $\operatorname{Hall}_{\pi}(N) \neq \emptyset$ in (2) can be avoided.

Finally, we investigate in Theorem 5 the structure of $N$ when the $G$-conjugacy class lengths of the considered vanishing elements in $G$ are prime powers. As a consequence of this study, when $N=G$, we obtain the next result.

Theorem D Let $G$ be a group. Assume that $\left|x^{G}\right|$ is a prime power for every vanishing element $x$ of $G$ of prime power order. Then, $G^{\prime}$ is nilpotent.

Other new interesting consequences arise from our contributions in the trivial case $N=G$ (see Sect. 5).

\section{Preliminaries}

The notation and terminology here are as follows. In the sequel, $p$ will be always a prime and $\pi$ will denote a set of primes. The set of prime divisors of the order of $G$ is $\pi(G)$. As usual, the set of all Sylow $p$-subgroups of $G$ is denoted by $\operatorname{Syl}_{p}(G)$, and $\operatorname{Hall}_{\pi}(G)$ is the set of all Hall $\pi$-subgroups of $G$. We write $\operatorname{Irr}(G)$ for the set of all irreducible complex characters of $G$. The set of vanishing elements of a group $G$ will be denoted by $\operatorname{Van}(G)$. CFSG means classification of finite simple groups. The remaining notation and terminology are standard in the framework of finite group theory, and we refer to the book [9] for details about character theory.

We gather some significant results for locating vanishing elements in a given group. As mentioned in "Introduction", it is elementary to see that a group is abelian if and only if every element is non-vanishing. In fact, this characterisation remains true, via the CFSG, when only prime power order elements are involved. 
Theorem 1 A group $G$ is abelian if and only if every prime power order element is nonvanishing in $G$.

Proof This is a direct application of [12, Theorem B], which asserts that a nonlinear irreducible character vanishes on some prime power order element.

Example 1 Concerning the above theorem, it is worth noting that, in general, it is not true that a normal subgroup $N$ is abelian if and only if every element of $N$ is non-vanishing in $G$, i.e. if $N \cap \operatorname{Van}(G)=\emptyset$ :

On the one hand, if $G=Q_{8}$ is a quaternion group of eight elements and $N$ is a normal subgroup of $G$ isomorphic to a cyclic group of order 4 , then $N$ is abelian and $N \cap \operatorname{Van}(G) \neq \emptyset$. On the other hand, by [10, Theorem 5.1], for any prime $p$ there exists a group $G$ having a normal non-abelian Sylow $p$-subgroup, and every $p$-element of $G$ is non-vanishing.

Proposition 1 [10, Theorem B] $G \backslash \mathbf{Z}(G)=\operatorname{Van}(G)$ for any nilpotent group $G$.

Observe that if a normal subgroup $N$ is nilpotent, then $N \backslash \mathbf{Z}(G)$ may not coincide with $\operatorname{Van}(G) \cap N$. For instance, one can consider as $G$ the normaliser in a Suzuki group of degree 8 of a Sylow 2-subgroup of it, and $N$ the Sylow 2-subgroup. It holds that $\operatorname{Van}(G) \cap N=\emptyset$ although clearly $N \backslash \mathbf{Z}(G) \neq \emptyset$.

Lemma 1 [8, Corollary 1.3] Let $H$ be a subgroup of a group $G$. Assume that $G=H \mathbf{C}_{G}(x)$ for some $x \in H$. Then, $x \in \operatorname{Van}(G)$ if and only if $x \in \operatorname{Van}(H)$.

The following four lemmas are crucial for proving Theorem 2, and the last two use the CFSG.

Lemma 2 [4, Lemma 5] Let $N$ be a minimal normal subgroup of $G$ so that $N=S_{1} \times \cdots \times S_{t}$, where $S_{i}$ is isomorphic to $S$, a non-abelian simple group. If $\sigma \in \operatorname{Ir}(S)$ extends to $\operatorname{Aut}(S)$, then $\sigma \times \cdots \times \sigma \in \operatorname{Irr}(N)$ extends to $G$.

Lemma 3 [11, Lemma 2.2] Let $G$ be a finite group, $p$ a prime, and $P \in \operatorname{Syl}_{p}(G)$. If $\chi \in \operatorname{Irr}(G)$ has $p$-defect zero, then $\chi$ is a constituent of $\left(1_{P}\right)^{G}$ and vanishes on the nontrivial $p$-elements of $G$.

Lemma 4 [11, Theorem 2.1] Let $S$ be a finite non-abelian simple group, $p$ a prime, and $P \in \operatorname{Syl}_{p}(S)$. Then, either $S$ has a p-defect zero character, or there exists a constituent $\theta \in \operatorname{Irr}(S)$ of the permutation character $\left(1_{P}\right)^{S}$ such that $\theta$ extends to $\operatorname{Aut}(S)$ and $\theta(x)=0$ for some p-element $x$ of $S$.

Lemma 5 [7, Lemma 2.8] Let $A$ be an abelian group that acts coprimely and faithfully by automorphisms on a group $M$. If $M$ is characteristically simple, then there exists $\theta \in \operatorname{Irr}(M)$ such that $I_{A}(\theta)=1$.

We also collect some preliminary results regarding conjugacy class sizes. We start with the next elementary properties which are frequently used, sometimes with no comment. 
Lemma 6 Let $N$ be a normal subgroup of a group $G$, and let p be a prime. We have:

(a) $\left|x^{N}\right|$ divides $\left|x^{G}\right|$, for any $x \in N$.

(b) $\left|(x N)^{G / N}\right|$ divides $\left|x^{G}\right|$, for any $x \in G$.

(c) If $x N \in G / N$ is a p-element, then $x N=y N$ for some p-element $y \in G$.

Lemma 7 Let $N$ be a normal subgroup of a group $G$, and let $H \in \operatorname{Hall}_{\pi}(N)$ for a set of primes $\pi$. If $x \in H$ is such that $\left|x^{G}\right|$ is a $\pi$-number, then $x$ lies in $\mathbf{O}_{\pi}(N)$.

Proof Since $\left|x^{N}\right|$ divides $\left|x^{G}\right|$, then $\left(\left|x^{N}\right|,|N: H|\right)=1$. It follows $N=H \mathbf{C}_{N}(x)$ and so $\left\langle x^{N}\right\rangle \leqslant \mathbf{O}_{\pi}(N)$.

Next we recall a generalisation of the above lemma when $N=G$ and $\pi=\{p\}$.

Lemma 8 [3, Lemma 3] Let $x \in G$. If $\left|x^{G}\right|$ is a power of a prime $p$, then $\left[x^{G}, x^{G}\right]$ is a p-group.

We end this section with the main result of [6], which will be necessary for proving Theorem 5. We present here an adapted version for our context of vanishing $G$-conjugacy classes.

Proposition 2 Let $G$ be a group which contains a non-trivial normal p-subgroup $N$, for a given prime $p$. Then, $\left|x^{G}\right|$ is a multiple of $p$ for each $x \in N \cap \operatorname{Van}(G)$.

\section{Proof of Theorems A and B}

Certainly, Theorem A is a direct application of Theorem B, so we focus on the proof of this last result. The next key proposition, which makes use of the CFSG, is inspired by the proof of [11, Theorem B].

Proposition 3 Let $M$ be a non-abelian minimal normal subgroup of a group $G$, and let $p$ be a prime divisor of $|M|$. Let $H$ be a subgroup of $G$ such that $H \cap M \in \operatorname{Syl}_{p}(M)$. Let $\beta \in \operatorname{Irr}(H / H \cap M)$ Then, there exists $\chi \in \operatorname{Irr}(G)$ such that $\chi$ is a constituent of $\beta^{G}$ and it vanishes on some p-element of $M$.

In particular, if $H=P \in \operatorname{Syl}_{p}(G)$, then there exists $\chi \in \operatorname{Irr}(G)$ such that $\chi$ is a constituent of $\left(1_{P}\right)^{G}$ and it vanishes on some p-element of $M$.

Proof We have $M=S_{1} \times \cdots \times S_{k}$, where all $S_{i}$ are isomorphic to a non-abelian simple group $S$ with $p \in \pi(S)$. If $\theta \in \operatorname{Irr}(S)$ is of $p$-defect zero, then $\eta=\theta \times \cdots \times \theta \in \operatorname{Irr}(M)$ and $\eta$ is also of $p$-defect zero. By Lemma 3 applied to $M$, we have $\left[\eta,\left(1_{H \cap M}\right)^{M}\right] \neq 0$ and $\eta$ vanishes on the non-trivial $p$-elements of $M$.

Since $\beta \in \operatorname{Irr}(H / H \cap M)$, we have $\left[\beta_{H \cap M}, 1_{H \cap M}\right] \neq 0$. Then, $\left(\beta^{H M}\right)_{M}=\left(\beta_{H \cap M}\right)^{M}=$ $\beta(1)\left(1_{H \cap M}\right)^{M}$ and $\left[\eta,\left(\beta^{H M}\right)_{M}\right]=\left[\eta^{H M}, \beta^{H M}\right] \neq 0$. Hence, there exists $\tau \in \operatorname{Irr}(H M)$ such that $\left[\tau, \eta^{H M}\right] \neq 0 \neq\left[\tau, \beta^{H M}\right]$. Let $\chi \in \operatorname{Irr}(G)$ over $\tau$. Then, $\chi_{M}$ is sum of $G$-conjugate characters of $\eta$. Therefore, $\chi$ vanishes on the non-trivial $p$-elements of $M$ and $\left[\chi, \beta^{G}\right]=\left[\chi_{H}, \beta\right] \neq 0$. 
Suppose now that $S$ does not have a character of $p$-defect zero. By Lemma 4 , there exists $\theta \in \operatorname{Irr}(S)$ such that $\left[\theta,\left(1_{H \cap S}\right)^{S}\right] \neq 0$ (note $\left.H \cap S \in \operatorname{Syl}_{p}(S)\right)$ which extends to $\operatorname{Aut}(S)$, and there exists a $p$-element $x \in S$ such that $\theta(x)=0$. Thus, $1 \neq y=(x, \ldots, x) \in M$ is a $p$-element and $\eta=\theta \times \cdots \times \theta$ vanishes on $y$, and certainly $\left[\eta_{H \cap M}, 1_{H \cap M}\right] \neq 0$. Since $\left[\beta_{H \cap M}, 1_{H \cap M}\right] \neq 0$, arguing as in the previous paragraph, we may affirm that there exists $\tau \in \operatorname{Irr}(H M)$ over $\eta$ and over $\beta$. Let $\chi \in \operatorname{Irr}(G)$ be over $\eta$, so $\left[\chi, \beta^{G}\right] \neq 0$. By Lemma $2, \eta$ extends to $G$. Let $\hat{\eta}$ be an extension of $\eta$. By Gallagher, $\chi=\hat{\eta} \rho$ for some $\rho \in \operatorname{Irr}(G / M)$. Therefore, $\chi$ lies over $\beta$ and $\chi(y)=\eta(y) \rho(1)=0$.

Theorem 2 Let $N$ be a normal subgroup of a group $G$, and let $P_{0}$ be a Sylow p-subgroup of $N$ for some prime $p$. Let $H$ be a subgroup of $G$ such that $H \cap N=P_{0}$, and let $\beta \in \operatorname{Irr}\left(H / P_{0}\right)$. Then, $P_{0}$ is normal in $N$ (and therefore in $G$ ) if and only if all irreducible constituents of $\beta^{G}$ do not vanish on any p-element of $N$.

Proof Suppose $P_{0} \unlhd N$. Let $\chi$ be a constituent of $\beta^{G}$ with $\beta \in \operatorname{Irr}(H / H \cap N)$. We have $\left[\beta_{P_{0}}, 1_{P_{0}}\right] \neq 0$, so $\left[\chi_{P_{0}}, 1_{P_{0}}\right] \neq 0$. Since $P_{0} \unlhd G$, then $\chi(x) \neq 0$ for all $p$-elements $x \in N$. Conversely, we consider that all irreducible constituents of $\beta^{G}$, where $\beta \in \operatorname{Irr}(H / H \cap N)$, do not vanish on any $p$-element of $N$, and we claim that $P_{0}$ is normal in $N$.

Suppose that the claim is false, and let us consider a counterexample which minimises $|G|$. Let $M$ be a minimal normal subgroup of $G$ such that $M \leqslant N$. We check that the hypotheses are inherited by $\bar{G}=G / M$. Certainly, $\bar{H} \cap \bar{N}=N / M \cap H M / M=(H \cap N) M / M \in \operatorname{Syl}_{p}(N / M)$. Since $\beta \in \operatorname{Irr}(H / H \cap N)$, then $\beta \in \operatorname{Irr}(H / H \cap M)$ so $\bar{\beta} \in \operatorname{Irr}(H M / M)$. Besides, $H \cap N \leqslant \operatorname{ker} \beta$ so $\overline{H \cap N} \leqslant \operatorname{ker} \bar{\beta}$. Let $\bar{\chi} \in \operatorname{Irr}(\bar{G})$ be an irreducible constituent of $\overline{\bar{\beta}}$ and $\bar{x} \in \bar{N}$ a $p$-element. Then, we may assume that $x \in N \backslash M$ is a $p$-element, and since $[\bar{\chi}, \bar{\beta} \bar{\beta}] \neq 0$, then it is easy to see that $\left[\chi_{H}, \beta\right] \neq 0$ and $\bar{\chi}(\bar{x})=\chi(x) \neq 0$. By minimality, we get $\overline{P_{0}} \unlhd \bar{G}$, so $P_{0} M \unlhd G$.

Let us assume that $p$ divides the order of $M$. If $M$ is a $p$-group, then $M \leqslant P_{0}$ and $P_{0}=P_{0} M \unlhd G$, a contradiction. Hence, $M$ is non-abelian. Since $\beta \in \operatorname{Irr}(H / H \cap M)$, in virtue of Lemma 3 there exists $\chi \in \operatorname{Irr}(G)$ such that $\left[\chi, \beta^{G}\right] \neq 0$ and $\chi(x)=0$ for some $p$-element $x \in M \leqslant N$, a contradiction again.

Thus, $p$ does not divide the order of $M$ and $\mathbf{O}_{p}(N)=1$. Let $K / M$ be a chief factor of $G$ such that $K \leq P_{0} M \unlhd G$, so $K / M$ is an abelian $p$-group. Note $K=M\left(K \cap P_{0}\right)$ and $K \cap P_{0} \in \operatorname{Syl}_{p}(K)$ is abelian. By Frattini's argument, $G=K \mathbf{N}_{G}\left(K \cap P_{0}\right)=M \mathbf{N}_{G}\left(K \cap P_{0}\right)$, so $\mathbf{C}_{K \cap P_{0}}(M) \unlhd G$ and $\mathbf{C}_{K \cap P_{0}}(M) \leqslant \mathbf{O}_{p}(N)=1$. Therefore, $K \cap P_{0}$ is an abelian $p$-group which acts coprimely and faithfully on $M$, and $M$ is characteristically simple. By Lemma 5 and Clifford theory, there exists $\theta \in \operatorname{Irr}(M)$ such that $\eta=\theta^{K}$ is irreducible. In particular, $\eta$ and all its conjugates vanish on $K \backslash M$. Therefore, if we prove that there exists $\chi \in \operatorname{Irr}(G)$ which lies over both $\eta$ and $\beta$, we will reach the final contradiction.

Let $T$ be the inertia subgroup for $\theta$ in $P_{0} M \unlhd G$. Since $(|T / M|,|M|)=1$, we have that $\theta$ extends to $\hat{\theta} \in \operatorname{Irr}(T)$ by [9, Corollary 6.28]. Further, $p$ does not divide $\hat{\theta}(1)$ so $\hat{\theta}_{P_{0} \cap T}$ has at least one linear constituent $\lambda$. As $T=M\left(P_{0} \cap T\right)$, then $P_{0} \cap T \cong T / M$ and we can see $\lambda$ also as a character of $T / M$. By Gallagher, $v=\bar{\lambda} \hat{\theta}$ is an irreducible character of $T$, where $\bar{\lambda}$ is the complex conjugate of $\lambda$. Moreover, $v_{M}=\theta$ and by Clifford correspondence $v^{P_{0} M} \in \operatorname{Irr}\left(P_{0} M\right)$. Hence, $\quad 0 \neq\left[1_{P_{0} \cap T}, \bar{\lambda}_{P_{0} \cap T} \hat{\theta}_{P_{0} \cap T}\right]=\left[1_{P_{0} \cap T}, v_{P_{0} \cap T}\right]=\left[\left(v_{P_{0} \cap T}\right)^{P_{0}}, 1_{P_{0}}\right]=$ $\left[\left(v^{P_{0} T}\right)_{P_{0}}, 1_{P_{0}}\right]=\left[\left(v^{P_{0} M}\right)_{P_{0}}, 1_{P_{0}}\right]=\left[\nu^{P_{0} M},\left(1_{P_{0}}\right)^{P_{0} M}\right]$. On theotherhand, $\left(\beta^{H N}\right)_{N}=\beta(1)\left(1_{P_{0}}\right)^{N}=$ $\beta(1)\left(\left(1_{P_{0}}\right)^{P_{0} M}\right)^{N}$, so $\left[\left(\beta^{H N}\right)_{N},\left(\nu^{P_{0} M}\right)^{N}\right]=\left[\left(\beta^{H N}\right)_{N}, \nu^{N}\right]=\left[\beta^{H N}, v^{H N}\right] \neq 0$. Therefore, there exists $\tau \in \operatorname{Irr}(H N)$ over $\beta$ and over $v$. Let $\chi \in \operatorname{Irr}(G)$ over $\tau$, so $\left[\chi, \beta^{G}\right] \neq 0$. Moreover, $\chi$ lies 
over $\theta$, and then $\chi$ lies over $\eta=\hat{\theta}$. Thus, $\chi_{K}$ is a sum of $G$-conjugate characters of $\eta$. Hence, $\chi(x)=0$ for all $x \in K \cap P_{0}$ and this is a final contradiction.

Theorem B in "Introduction" is now a corollary of the above result when we take $H$ a Sylow $p$-subgroup of $G$ [for Theorem B(iii)] and $H=P_{0}$ [for Theorem B(ii)]. Moreover, when $N=G$ in Theorem B, then we obtain the characterisation (i)-(ii).

Theorem 3 [11, Theorem B] Let $G$ be a group, $p$ a prime number, and P a Sylow p-subgroup of $G$. Then the following conditions are equivalent:

(i) $P$ is normal in $G$.

(ii) $\chi(x) \neq 0$ for all irreducible constituents $\chi$ of $\left(1_{P}\right)^{G}$ and all $x \in P$.

(iii) $p$ does not divide $\chi(1)$ for all irreducible constituents $\chi$ of $\left(1_{P}\right)^{G}$.

\section{Example 2}

(1) Note that in Theorem B we can have $\beta \in \operatorname{Irr}\left(P / P_{0}\right)$ distinct from $1_{P}$, in contrast to Theorem 3: Let $G$ be a symmetric group of degree 4 and let $N$ be an alternating group of degree 4. Take $P \in \operatorname{Syl}_{2}(G)$. Then, there exists a non-trivial irreducible character $\beta \in \operatorname{Irr}(P)$ with $P_{0}=P \cap N \leqslant \operatorname{ker} \beta$. Additionally, the irreducible constituents of $\beta^{G}$ do not vanish on the $p$-elements of $N$, so the hypotheses in Theorem B(iii) are fulfilled.

(2) The following equivalence, similar to Theorem 3(i)-(iii), is not true: $P_{0}$ is a normal Sylow $p$-subgroup of $N$ if and only if $p$ does not divide $\chi(1)$ for all irreducible constituents of $\left(1_{P_{0}}\right)^{G}$ : Consider $G$ and $N$ as above. Then, $\left(1_{P_{0}}\right)^{G}$ has three distinct irreducible constituents, being one of them of degree 2 .

Both examples have been checked using the software GAP [13].

Let consider now a set of primes $\pi$ instead of a single prime $p$. As a consequence of Theorem A, we give in the following proposition extra information on the structure of a $\pi$ -complement of $G$ when $N$ contains a Hall $\pi$-subgroup of it.

Proposition 4 Let $N$ be a normal subgroup of a group $G$ such that every prime power order $\pi$-element of $N$ is non-vanishing in $G$, for a set of primes $\pi$. Then, $N$ has a nilpotent normal Hall $\pi$-subgroup.

Further, if $|G: N|$ is a $\pi^{\prime}$-number, then any $\pi$-complement $F$ of $G$ verifies that $F \mathbf{Z}(G)$ is self-normalising.

Proof Certainly, in virtue of Theorem A we have that $N$ has a nilpotent normal Hall $\pi$ -subgroup, say $H$. In fact, if $|G: N|$ is not divisible by any prime in $\pi$, then $H$ is a normal Hall $\pi$-subgroup of $G$. Let $F$ be a $\pi$-complement of $H$ in $G$, so $G=H F$. We aim to show that $F \mathbf{Z}(G)=\mathbf{N}_{G}(F \mathbf{Z}(G))$. Take a prime power order element $x \in \mathbf{N}_{H}(F \mathbf{Z}(G))$. Then, $F^{x} \mathbf{Z}(G)=(F \mathbf{Z}(G))^{x}=F \mathbf{Z}(G)$, so there exists some $y \in F \mathbf{Z}(G)$ such that $F^{x}=F^{y}=F$. Thus, $x \in \mathbf{N}_{H}(F) \leqslant \mathbf{C}_{H}(F)$ because $\left[\mathbf{N}_{H}(F), F\right] \leqslant H \cap F=1$. Therefore, $G=H F=H \mathbf{C}_{G}(x)$. Since $x \notin \operatorname{Van}(G)$ by assumption, then Lemma 1 yields that $x \notin \operatorname{Van}(H)$. Now Proposition 1 applies because $H$ is nilpotent, so $x \in \mathbf{Z}(H) \cap \mathbf{C}_{G}(F) \leqslant \mathbf{Z}(G)$. As this argument is valid for every prime power order element in $\mathbf{N}_{H}(F \mathbf{Z}(G))$, then $\mathbf{N}_{H}(F \mathbf{Z}(G)) \leqslant \mathbf{Z}(G)$. Finally, note that $\mathbf{N}_{G}(F \mathbf{Z}(G))=\mathbf{N}_{G}(F \mathbf{Z}(G)) \cap H F=F\left(\mathbf{N}_{H}(F \mathbf{Z}(G))\right)=F \mathbf{Z}(G)$, as wanted. 
Corollary 1 Let $G$ be a group such that all the p-elements are non-vanishing. Then, $G$ has a normal Sylow p-subgroup, and $F \mathbf{Z}(G)$ is self-normalising for any p-complement $F$ of $G$.

\section{Lengths of G-conjugacy classes of vanishing elements}

We start by showing an extension of Lemma 8 for a set of primes $\pi$ and a $G$-conjugacy class. The proof is inspired by [1, Theorem C] under the weaker hypothesis of the $\pi$ -separability of the normal subgroup $N$.

Proposition 5 Let $N$ be a normal $\pi$-separable subgroup of a group $G$. If $x \in N$ is such that $\left|x^{G}\right|$ is a $\pi$-number, then $\left[x^{G}, x^{G}\right] \leqslant \mathbf{O}_{\pi}(N)$. In particular, $x \mathbf{O}_{\pi}(N) / \mathbf{O}_{\pi}(N) \in \mathbf{Z}\left(\mathbf{F}\left(N / \mathbf{O}_{\pi}(N)\right)\right)$.

Indeed, if $\pi$ consists of a single prime $p$, then the same statement is valid even if $N$ is not p-soluble.

Proof In order to prove the first claim, let us consider a counterexample which minimises $|G|+|N|$. One can clearly assume $\mathbf{O}_{\pi}(N)=1$, so we aim to get the contradiction $\left[x^{G}, x^{G}\right]=1$. Let us suppose firstly that $\langle x\rangle$ is subnormal in $G$. Then, $x \in \mathbf{F}(G)$. As $\mathbf{F}(G)$ is a $\pi^{\prime}$-group and $\left|x^{G}\right|$ is a $\pi$-number, then clearly $x \in \mathbf{Z}(\mathbf{F}(G))$ and $\left\langle x^{G}\right\rangle \leqslant \mathbf{Z}(\mathbf{F}(G))$, so $\left[x^{G}, x^{G}\right]=1$.

Next we assume that the normal subgroup $M:=\left\langle x^{G}\right\rangle$ is proper in $N$. Then, by minimality we obtain $\left[x^{M}, x^{M}\right]=1$, and it follows that $x \in \mathbf{Z}\left(\left\langle x^{M}\right\rangle\right)$. In particular, $\langle x\rangle$ is subnormal in $M$, and therefore in $G$, which contradicts the previous paragraph. Hence, $M=N$.

Let $K:=\mathbf{O}_{\pi^{\prime}}(N)$. Since $N$ is $\pi$-separable, then $K$ is non-trivial. It follows from the class size hypothesis that $K$ centralises $x^{G}$, so $K$ is central in $N=\left\langle x^{G}\right\rangle$. As $\left[x^{G}, x^{G}\right] K / K \leqslant \mathbf{O}_{\pi}(N / K)$ by minimality, and $\mathbf{O}_{\pi}(N / K)=\mathbf{O}_{\pi}(N) K / K$ because $K$ is central $N$, we deduce $\left[x^{G}, x^{G}\right]=N^{\prime} \leqslant K \leqslant \mathbf{Z}(N)$. Therefore, $N$ is a nilpotent $\pi^{\prime}$-group. Since $\left|x^{G}\right|$ is a $\pi$-number, we obtain $x \in \mathbf{Z}(N)$ and $\left[x^{G}, x^{G}\right]=1$.

Next, we concentrate on the second assertion. Let $\bar{G}:=G / \mathbf{O}_{\pi}(N)$. Then, $\left[\bar{x}^{\bar{G}}, \bar{x}^{\bar{G}}\right]=1$ by the first claim. It follows that $\langle\bar{x}\rangle \unlhd \mathbf{Z}(\langle\bar{x} \bar{G}\rangle) \unlhd \bar{G}$, so $\langle\bar{x}\rangle \leqslant \mathbf{F}(\bar{G}) \cap \bar{N} \leqslant \mathbf{F}(\bar{N})$. As $\mathbf{F}(\bar{N})$ is a normal $\pi^{\prime}$-subgroup of $\bar{G}$ and $|\bar{x} \bar{G}|$ is a $\pi$-number, then necessarily $\bar{x} \in \mathbf{Z}(\mathbf{F}(\bar{N}))$.

Finally, observe that the last statement follows from Lemma 8, since $\left[x^{G}, x^{G}\right] \leqslant \mathbf{O}_{p}(G) \cap N \leqslant \mathbf{O}_{p}(N)$.

Example 3 Note that the $\pi$-separability assumption in the previous result cannot be removed, even when $N=G$ : Consider any non-trivial element in the centre of a Sylow $p$-subgroup of a non-abelian simple group and $\pi=p^{\prime}$, for a prime divisor $p$ of its order.

For a normal subgroup $N$ of a group $G$, note that if $x N$ is a vanishing (prime power order) element of $G / N$, then we can assume that $x$ is also a vanishing (prime power order) element of $G$. This is because there exists a bijection between $\operatorname{Irr}(G / N)$ and the set of all characters in $\operatorname{Irr}(G)$ containing $N$ in their kernel. This fact will be used in the sequel with no reference.

As an application of the above proposition and mainly Theorem A, we prove Theorem C in "Introduction". 
Proof of Theorem C (1) Assume that $N$ is $\pi$-separable and that $\left|x^{G}\right|$ is a $\pi^{\prime}$-number for every prime power order $\pi$-element $x \in \operatorname{Van}(G) \cap N$. Let us prove that $N / \mathbf{O}_{\pi^{\prime}}(N)$ has a normal Sylow $p$-subgroup for each prime $p \in \pi$. Certainly, whenever $\mathbf{O}_{\pi^{\prime}}(N) \neq 1$, the assertion follows by induction, considering the groups $G / \mathbf{O}_{\pi^{\prime}}(N)$ and $N / \mathbf{O}_{\pi^{\prime}}(N)$. Therefore, we may assume that $\mathbf{O}_{\pi^{\prime}}(N)=1$. Let $Z_{p}:=\mathbf{Z}\left(\mathbf{O}_{p}(N)\right)$. In virtue of Proposition 5, we have that all the $p$-elements of $\operatorname{Van}(G) \cap N$ lie in $\mathbf{Z}(\mathbf{F}(N))$, and thus, in $Z_{p}$. Therefore, if we denote $\bar{G}:=G / Z_{p}$, then it follows that no prime power order $p$-element of $\bar{N}$ is vanishing in $\bar{G}$. Now Theorem A yields that $\bar{N}$ has a normal Sylow $p$-subgroup $\bar{P}$, where $P \in \operatorname{Syl}_{p}(N)$. Since $Z_{p}$ is a $p$-group, then $P$ is normal in $N$ clearly and we get the claim. As this is valid for each prime $p \in \pi$, then $N / \mathbf{O}_{\pi^{\prime}}(N)$ has a nilpotent normal Hall $\pi$-subgroup, as wanted.

(2) Assume that $N$ has Hall $\pi$-subgroups and that $\left|x^{G}\right|$ is a $\pi$-number for every prime power order $\pi$-element $x \in \operatorname{Van}(G) \cap N$. We claim that $N$ has a normal Hall $\pi$-subgroup. Clearly, we may assume $\mathbf{O}_{\pi}(N)=1$. Let $H \in \operatorname{Hall}_{\pi}(N)$, and let $p \in \pi$. If $x \in N \cap \operatorname{Van}(G)$ is a $p$-element, then $x \in P \in \operatorname{Syl}_{p}(N)$. Hence, there exists $g \in N$ such that $x^{g} \in P^{g} \in \operatorname{Syl}_{p}(H)$. Now Lemma 7 yields $x^{g} \in \mathbf{O}_{\pi}(N)=1$. Thus, there are no $p$-elements in $N \cap \operatorname{Van}(G)$, and by Theorem A, we get that $N$ has a normal Sylow $p$-subgroup. Since this is valid for every prime $p \in \pi$, then $N$ has a (nilpotent) normal Hall $\pi$-subgroup, as desired.

Next, we show that $N$ has nilpotent Hall $\pi^{\prime}$-subgroups under the additional assumption that the prime power order $\pi^{\prime}$-elements in $N \cap \operatorname{Van}(G)$ have also $G$-class sizes not divisible by any prime in $\pi^{\prime}$. Note that $N$ is $\pi$-separable because it has a normal Hall $\pi$-subgroup, say $H$. If we take any prime power order element $x H \in(N / H) \cap \operatorname{Van}(G / H)$, then we may suppose that $x \in N \cap \operatorname{Van}(G)$ is a prime power order element, so by assumptions $\left|x^{G}\right|$ is a $\pi$ -number. Thus, $\left|(x H)^{G / H}\right|$ is also a $\pi$-number. Therefore, every $\left|(x H)^{G / H}\right|$ is a $\pi$-number for each prime power order $\pi^{\prime}$-element $x H \in(N / H) \cap \operatorname{Van}(G / H)$, so by assertion (1) the $\pi^{\prime}$ -group $N / H$ is nilpotent. Since $N / H$ is isomorphic to a Hall $\pi^{\prime}$-subgroup of $N$, the proof is completed.

Example 4 We remark that the $\pi$-separability assumption in Theorem $\mathrm{C}(1)$ is necessary for the first claim. Let $G$ be a symmetric group of degree 5, and let $N$ be an alternating group of degree 5. Consider $\pi=\{3\}$. Then, all the 3-elements in $N \cap \operatorname{Van}(G)$ have conjugacy class size equal to 20 . Nevertheless, $N / \mathbf{O}_{\pi^{\prime}}(N)=N$ does not have a normal Sylow 3-subgroup.

Example 5 It is not difficult to find groups satisfying the assumptions of Theorem $\mathrm{C}$. For instance, let $G=A \Gamma\left(2^{3}\right)$ be an affine semilinear group of order 168, and let $N$ be the Hall $3^{\prime}$-subgroup of $G$. If we consider $\pi=\{7\}$, then the pair $(N, G)$ satisfies the hypotheses of Theorem $\mathrm{C}(1)$. Concerning Theorem $\mathrm{C}(2)$, if $\pi$ is any set of prime numbers, $G=\mathbf{O}_{\pi}(G) \times \mathbf{O}_{\pi^{\prime}}(G)$ and $N=\mathbf{O}_{\pi}(G)$, then the pair $(N, G)$ certainly holds the hypotheses.

The next theorem combines the arithmetical conditions of Theorem $\mathrm{C}$ on the vanishing $G$-class sizes.

Theorem 4 Let $N$ be a normal $\pi$-separable subgroup of a group $G$. Assume that $\left|x^{G}\right|$ is either a $\pi$-number or a $\pi^{\prime}$-number for every prime power order $\pi$-element $x \in \operatorname{Van}(G) \cap N$. Then, $N / \mathbf{O}_{\pi^{\prime}}(N)$ has a normal Hall $\pi$-subgroup. Thus, $N$ has $\pi$-length at most 1.

Proof First, we claim that $O:=\mathbf{O}_{\pi, \pi^{\prime}}(N)$ contains a Sylow $p$-subgroup of $N$, for a prime $p \in \pi$. Let $x \in \operatorname{Van}(G) \cap N$ be a $p$-element. If $\left|x^{G}\right|$ is a $\pi$-number, then $x$ lies in $\mathbf{O}_{\pi}(N)$ 
because of Lemma 7, so clearly $x \in O$. If $\left|x^{G}\right|$ is a $\pi^{\prime}$-number, then by Proposition 5 we get $x \mathbf{O}_{\pi^{\prime}}(N) \in \mathbf{F}\left(N / \mathbf{O}_{\pi^{\prime}}(N)\right)$, and again $x$ lies in $O$. It follows that $\bar{N}:=N / O$ contains no vanishing $p$-element of $G / O$, so $\bar{N}$ has a normal Sylow $p$-subgroup $\bar{P}$ in virtue of Theorem A. Since $p \in \pi$ and clearly $\mathbf{O}_{\pi}(\bar{N})=1$, thus $\bar{P}=1$.

Therefore, $O$ contains a Sylow $p$-subgroup of $N$ for every $p \in \pi$, and thus, $O / \mathbf{O}_{\pi^{\prime}}(N)$ is a Hall $\pi$-subgroup of $N / \mathbf{O}_{\pi^{\prime}}(N)$.

The main theorem of [1] examines groups such that all their $\pi$-elements have prime power class sizes. The next result is a "vanishing version" of that theorem for prime power order elements and in the context of $G$-conjugacy classes.

Theorem 5 Let $N$ be a normal subgroup of a group $G$. Assume that $\left|x^{G}\right|$ is a prime power for each prime power order $\pi$-element $x \in N$ that is vanishing in $G$. Then $N / \mathbf{O}_{\pi^{\prime}}(\mathbf{F}(N))$ has a normal Hall $\pi$-subgroup.

In particular, if $\pi$ is the set of prime divisors of $|N|$, then $N / \mathbf{F}(N)$ is nilpotent.

Proof We claim that $\bar{N}:=N / \mathrm{F}(N)$ has a normal Hall $\pi$-subgroup, and therefore, $N / \mathbf{O}_{\pi^{\prime}}(\mathbf{F}(N))$ so does because $\mathbf{F}(N) / \mathbf{O}_{\pi^{\prime}}(\mathbf{F}(N))$ is a $\pi$-group. Arguing by contradiction, and in virtue of Proposition 4, we may assume that $\bar{N} \cap \operatorname{Van}(\bar{G})$ contains a non-trivial $q$-element for some prime $q \in \pi$, say $\bar{x}$. Hence, we may suppose that $x \in(N \cap \operatorname{Van}(G)) \backslash \mathbf{F}(N)$ is a $q$-element. By assumptions, we have that $\left|x^{G}\right|$ is a power of some prime $p$. Observe that, since $x \notin \mathbf{F}(N)$, then $q \neq p$ due to Lemma 7. Now the last statement of Proposition 5 yields $\left(\left\langle x^{G}\right\rangle\right)^{\prime} \leqslant \mathbf{O}_{p}(N) \leqslant \mathbf{F}(N)$, so $\overline{\langle x\rangle}$ is a subnormal nilpotent subgroup of $\bar{N}$. It follows that $\bar{x} \in \mathbf{F}(\bar{N})$, and as $\bar{x}$ is a $q$-element, then $\bar{x} \in \mathbf{O}_{q}(\bar{N})$. Now $\left|\bar{x}^{\bar{G}}\right|$ is a multiple of $q$ by Proposition 2 , and then $\left|x^{G}\right|$ so is, a contradiction.

Finally, if $\pi=\pi(N)$, then with a similar argument we deduce that there is no prime power order element in $N / \mathbf{F}(N)$ vanishing in $G / \mathbf{F}(N)$. Hence, Theorem A applies and $N / \mathbf{F}(N)$ is nilpotent.

\section{Some consequences on vanishing conjugacy classes}

New interesting contributions on the lengths of vanishing classes of a group $G$ emerge from Theorems C, 4 and 5 when $N=G$.

Theorem 6 Let $G$ be a $\pi$-separable group. If $\left|x^{G}\right|$ is a $\pi^{\prime}$-number for every prime power order $\pi$-element $x \in \operatorname{Van}(G)$, then $G / \mathbf{O}_{\pi^{\prime}}(G)$ has a nilpotent normal Hall $\pi$-subgroup. Therefore, $G$ has nilpotent Hall $\pi$-subgroups, and its $\pi$-length is at most 1.

Theorem 7 Let $G$ be a finite group such that $\operatorname{Hall}_{\pi}(G) \neq \emptyset$. Assume $\left|x^{G}\right|$ is a $\pi$-number for every prime power order $\pi$-element $x \in \operatorname{Van}(G)$. Then $G$ has a normal Hall $\pi$-subgroup.

Further, if the prime power order $\pi^{\prime}$-elements in $\operatorname{Van}(G)$ have also class size a $\pi$-number, then the Hall $\pi^{\prime}$-subgroups of $G$ are nilpotent. 
Theorem 8 Let $G$ be a group. Suppose that $\left|x^{G}\right|$ is either a $\pi$-number or a $\pi^{\prime}$-number for every prime power order $\pi$-element $x \in \operatorname{Van}(G)$. Then $G / \mathbf{O}_{\pi^{\prime}}(\mathbf{F}(G))$ has a normal Hall $\pi$ -subgroup. In particular, $G$ has $\pi$-length at most 1 .

Proof of Theorem $D$ Arguing as in the proof of Theorem 5, we can see that $G / \mathbf{F}(G)$ has no prime power order vanishing elements. Thus, Theorem 1 applies and $G / \mathbf{F}(G)$ is abelian, so $G^{\prime}$ is nilpotent.

Acknowledgements This research has been carried out during a stay of the second author at the Instituto Universitario de Matemática Pura y Aplicada (IUMPA-UPV) of the Universitat Politècnica de València. He wishes to thank the members of the IUMPA for their hospitality. The authors would like to thank G. Navarro for useful conversations during the preparation of the paper.

Funding The first author is supported by Proyecto Prometeo II/2015/011, Generalitat Valenciana (Spain). The research of the second author is partially funded by the Istituto Nazionale di Alta Matematica - INdAM. The third author acknowledges the predoctoral grant ACIF/2016/170, Generalitat Valenciana (Spain). The first and third authors are also supported by Proyecto PGC2018-096872-B-I00, Ministerio de Ciencia, Innovación y Universidades (Spain).

\section{References}

1. Beltrán, A., Felipe, M.J.: Prime powers as conjugacy class lengths of $\pi$-elements. Bull. Aust. Math. Soc. 69, 317-325 (2004)

2. Beltrán, A., Felipe, M.J., Malle, G., Moretó, A., Navarro, G., Sanus, L., Solomon, R., Tiep, P.H.: Nilpotent and abelian Hall subgroups in finite groups. Trans. Am. Math. Soc. 368, 2497-2513 (2016)

3. Berkovich, Y., Kazarin, L.S.: Indices of elements and normal structure of finite groups. J. Algebra 283, 564-583 (2005)

4. Bianchi, M., Chillag, D., Lewis, M.L., Pacifici, E.: Character degree graphs that are complete graphs. Proc. Am. Math. Soc. 135, 671-676 (2007)

5. Brough, J., Kong, Q.: On vanishing criteria that control finite group structure II. Bull. Aust. Math. Soc. 98, 251-257 (2018)

6. Brough, J.: Non-vanishing elements in finite groups. J. Algebra 460, 387-391 (2016)

7. Dolfi, S., Pacifici, E., Sanus, L., Spiga, P.: On the orders of zeros of irreducible characters. J. Algebra 321, 345-352 (2009)

8. Grüninger, M.: Two remarks about non-vanishing elements in finite groups. J. Algebra 460, 366-369 (2016)

9. Isaacs, I.M.: Character Theory of Finite Groups. Academic Press Inc., London (1976)

10. Isaacs, I.M., Navarro, G., Wolf, T.R.: Finite group elements where no irreducible character vanishes. J. Algebra 222, 413-423 (1999)

11. Malle, G., Navarro, G.: Characterizing normal Sylow $p$-subgroups by character degrees. J. Algebra 370, 402-406 (2012)

12. Malle, G., Navarro, G., Olsson, J.B.: Zeros of characters of finite groups. J. Group Theory 3, 353-368 (2000)

13. The GAP Group: GAP_Groups, Algorithms, and Programming. Version 4.10.0 (2018). http://www. gap-system.org

Publisher's Note Springer Nature remains neutral with regard to jurisdictional claims in published maps and institutional affiliations. 